\title{
THE EXTRAGALACTIC X-RAY AND $\gamma$-RAY BACKGROUNDS
}

\author{
G. Setti \\ European Southern Observatory \\ Karl-Schwarzschild-Str. 2 \\ D-0846 Garching bei München \\ FRG
}

\begin{abstract}
The long-standing problem of the origin of the extragalactic X-ray background (XRB) is reviewed. Although the shape of the spectrum in the 3-100 keV interval is suggestive of an optically thin bremsstrahlung at $\sim 40 \mathrm{keV}$, the interpretation in terms of a hot intergalactic gas (IGG) requires a rather extreme energy supply and a gas density conflicting with the baryon density upper limit derived from primordial nucleosynthesis calculations in the standard hot big-bang model. A summary discussion of the estimated contributions from the integrated X-ray emission of known classes of extragalactic discrete sources at a reference energy of $2 \mathrm{keV}$ is given. Although these estimates are still uncertain, the subtraction of a "minimum" contribution drastically modifies the $40 \mathrm{keV}$ thermal shape, which is the prima facie evidence of a hot IGG. AGNs are the main contributors. Low luminosity AGNs (Seyfert type 1 nuclei) at redshift $z=1-2$ may in fact saturate the $2 \mathrm{keV} \mathrm{XRB}$, but their observed hard X-ray spectra are on the average unlike (much too steep) that of the XRB. This has led a number of authors to postulate new classes of sources and some exotic models which are briefly summarized. However, if a recently proposed unified scheme of AGNs holds, then the bulk of the XRB intensity can be explained independently of the observed spectral differences and with a mild cosmological evolution. The origin of the extragalactic $\gamma$-ray background is briefly commented upon in the concluding remarks.
\end{abstract}

\section{INTRODUCTION}

The spectrum of the extragalactic components of the $X$ - and $\gamma$-ray backgrounds for energies $\geq 3 \mathrm{keV}$ is reproduced in Figure 1. Its overall extent covers almost five decades in energy.

The cosmic X-ray background (XRB) has been the subject of many reviews (e.g., Boldt, 1987). Here we summarize the main characteristics of the observed radiation flux in the interval $3 \mathrm{keV}$ to several $100 \mathrm{keV}$. At lower energies the spectrum of the XRB is essentially unknown because the galactic emission begins to be substantial and difficult to separate. It is well known that in the energy interval 3-100 keV the data are well fitted by an optically thin thermal bremsstrahlung spectrum at a temperature $\mathrm{kT} \cong 40 \mathrm{keV}$, while at higher energies up to several hundred $\mathrm{keV}$ the energy spectrum can be represented by a power law of index $\alpha \cong$ -1.8 (Gruber et al., 1984). It is useful to note that at low photon energies, say below $\sim 20$ $\mathrm{keV}$, the energy spectrum is consistent with a power law of index $\alpha \cong-0.4$, much flatter than the typical spectra of known classes of extragalactic X-ray sources, a fact that, as will be seen later, plays a key role in the overall discussion on the origin of the XRB.

The intensity integrated over the spectrum corresponds to a present energy density in the radiation field of $\cong 5 \times 10^{-5} \mathrm{eV} \mathrm{cm}^{-3}$, of which about $2 / 3$ is below $40 \mathrm{keV}$. Although this value is not large in absolute terms compared to what is found in other regions of the electromagnetic spectrum, it remains unexplained why so much energy has been channeled in high energy photons. In fact, from the beginning it was clear that the bulk of the XRB must have been produced at higher redshifts either as a result of diffuse processes taking place in the intergalactic spacer and/or as the superposition of the emission from discrete sources. 


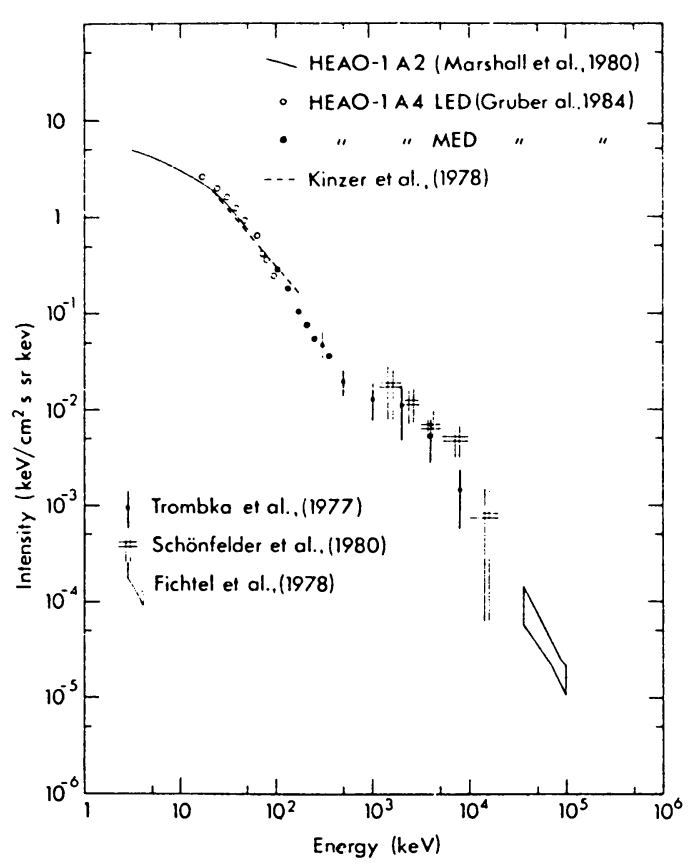

Figure 1. The differential energy spectrum of the extragalactic $\mathrm{X}$ - and $\boldsymbol{\gamma}$-ray backgrounds.

The large scale distribution of the intensity of the XRB is highly isotropic when a (small) anisotropic component ( $\leq 2 \%$ at high galactic latitudes) due to the emission from the Galaxy is removed. There is also a dipole component with a fractional amplitude of $5 \times 10^{-3}$ which, if interpreted in terms of the Compton-Getting effect, gives an observer's velocity vector (Shafer, 1983; Shafer and Fabian, 1983) consistent within the error with that inferred from the cosmic microwave background dipole anisotropy (Smoot et al., 1977; Cheng et al., 1979). This is a tentative result since rms fluctuations relative to large solid angles is $\gtrsim 3 \times 10^{-3}$ (Boldt, 1988). These findings support the idea of a cosmological origin of the XRB.

Of particular interest to us here are the measurements of the small-scale intensity fluctuations which may be directly related to the statistical fluctuations in the source populations contributing to the XRB and their clustering properties and/or to the clumpiness of the medium if diffuse emission is important.

At energies $\geq 3 \mathrm{keV}$ the surface brightness fluctuations are $\approx 0.02$ on angular scales of several degrees (Shafer, 1983). These fluctuations are consistent with an extrapolation of the number counts (N) vs. flux (f) relationship with an Euclidean power law of the form $N(>f) \alpha f^{-1.5}$ down to a flux about one order of magnitude fainter than the survey limit of the bright sources found in the HEAO-1 complete sample. It is also found that any population of sources which would contribute most of the XRB should have a surface density $\gtrsim 50$ sources $\operatorname{deg}{ }^{-2}$.

In the $1-3 \mathrm{keV}$ energy band, arcminute scale fluctuations of the XRB have been analyzed by Hamilton and Helfand (1987) using deep survey fields obtained by the Einstein Observatory. The measured granularity requires that the $\mathrm{X}$-ray source counts extend well below the High Sensitivity Survey limit of the Einstein Observatory. However, if a population of discrete sources had to account for most of the XRB in this energy band, then the source 
counts should flatten well below the Euclidean slope and the derived surface density of such sources should be no less than $\sim 5 \times 10^{3} \mathrm{deg}^{-2}$. These results are confirmed by an independent analysis of other Einstein Observatory deep fields (Barcons, 1989, this volume).

Going from the hard X-rays into the $\gamma$-ray band the spectrum starts to flatten. Measurements at these energies are difficult because they are affected by local backgrounds produced by cosmic rays. However, two different experiments whose results are reproduced in Figure 1 seem to confirm consistently the presence of a spectral hump at about $2 \mathrm{MeV}$, while very little is known about the spatial isotropy of the observed flux. At even higher energies $(35-200$ $\mathrm{MeV}$ ), the basic information comes from the SAS-2 satellite. Here it is difficult to separate out the contribution from the Galaxy, which is very substantial. The derived extragalactic flux is generally consistent with an extrapolation of the spectrum from lower energies (see also Thompson and Fichtel, 1982), while the large-scale isotropy cannot be determined to better than $\sim 30 \%$.

In view of the above the major part of this review will be dedicated to a discussion of the origin of the XRB.

\section{THE XRB AND A HOT INTERGALACTIC MEDIUM}

The fact that the observed spectrum of the XRB between 3 and $100 \mathrm{keV}$ can be so well approximated by an optically thin bremsstrahlung has renewed an interest in the proposal of Cowsik and Kobetich (1972) that the XRB is due to a hot diffuse intergalactic gas (IGG) with a (present) temperature of $\sim 5 \times 10^{8} \mathrm{~K}$ (Field and Perrenod, 1977). Because of the relative inefficiency of the bremsstrahlung mechanism, the cooling time is much larger than the Hubble time for any reasonable value of the baryon density parameter and, as a result, the energy deposited in the gas is comparable to that in the cosmic microwave background (CMB). It is difficult to understand how this huge amount of energy could be supplied by the galaxies which on the average would be required to deposit $\approx 10^{64}$ ergs each into the IGG. For this reason, one has relegated the reheating of the IGG to earlier epochs, corresponding to a redshift $z \sim 3$, for which there are indications that nonthermal explosive phenomena in galaxies were much more frequent and powerful as shown by the dramatic cosmological evolution associated with quasars and radio galaxies. At the same time, since the thermal bremsstrahlung emission depends on the square of the gas density, the energy requirements would also be somewhat alleviated.

Subsequent work has simply assumed that the IGG is suddenly heated up at a redshift $z_{m}$, and then cools down because of the expansion and Compton losses with the photons of the CMB. Compton cooling starts to dominate progressively at redshift $z>3.5$ and therefore it becomes energetically unfavorable to assume that the IGG has been reheated at the required temperature at even larger redshifts (Guilbert and Fabian, 1986). Moreover, these authors point out that the low energy spectrum from a Compton cooled optically thin gas would be much steeper $(0.5<\alpha<1)$ than the observed XRB. Therefore, in order to fit the shape of the XRB spectrum, the gas should have been reheated at a temperature $T \approx\left(1+z_{m}\right) 40 \mathrm{keV}$, where $z_{m}=3-4$, and relativistic corrections to the bremsstrahlung emission and to the gas thermodynamics must be taken into account (Barcons and Lapiedra, 1985; Guilbert and Fabian, 1986; Barcons, 1987). Even with the enhanced emission due to the relativistic corrections, such as electron-electron bremsstrahlung, it turns out that, in order to meet the XRB intensity, the IGG density should correspond to $\Omega_{I G G}>0.2$ (the density parameter $\Omega=\rho / \rho_{c}$, where $\rho_{c}=2 \times 10^{29} h_{0}^{2} g \mathrm{~cm}^{-3}$ is the closure density of the universe with $h_{0}=$ Hubble constant in 
units of $100 \mathrm{~km} \mathrm{~s}^{-1} \mathrm{Mpc}^{-1}$. A recent discussion (Taylor and Wright, 1989) indicates $\Omega_{I G G} \approx 0.276$ for a model in which the IGG was heated at $z_{m}=5$ so that its energy content reached $\sim 50 \%$ of that in the CMB and then cooled down to a present temperature of $\sim 10$ $\mathrm{keV}$. This is an additional difficulty with the hot IGG interpretation of the XRB in the framework of the standard hot big-bang model of the universe, because the requirement that the primordial nucleosynthesis produces the observed abundances of light elements, in particular deuterium, places an upper bound of $\Omega_{B}<0.20$ to the total density of baryonic matter (Boesgaard and Steigman, 1985; Reeves et al., 1989).

Since the bremsstrahlung emission is proportional to the square of the gas density, a way out of the above difficulty would be to assume that the IGG is clumped, thereby reducing the mean density required to fit the XRB intensity (Field and Perrenod, 1977; Guilbert and Fabian, 1986). Then, one would have to assume a two-phase model of the IGG in which hot, relatively dense clumps of gas responsible for the observed XRB between 3 and $100 \mathrm{keV}$ are in pressure equilibrium with a surrounding thinner but hotter gas. Obviously, the observed surface brightness fluctuations in the XRB can be used to constrain the physical parameters of this two-phase model of the IGG, but much more stringent constraints are imposed from the analysis of the temperature fluctuations of the CMB induced by Compton scattering the photons out of the Rayleigh-Jeans portion of the CMB spectrum (Guilbert and Fabian, 1986). In a recent work Barcons and Fabian (1988) come to the conclusion that the size of the clumps should be less than a few tens of $\mathrm{kpc}$, not to exceed the upper limits obtained with the VLA (Fomalont et al., 1984; Knoke et al., 1984) on the fluctuations of the CMB temperature on sub-arcmin angular scales. If this result holds, it is certainly not easy to imagine how this fine-structured two-phase plasma could have originated and maintained, and the whole idea of explaining the XRB by an optically thin bremsstrahlung seems to meet very serious difficulties.

In this discussion we have implicitly assumed that the integrated contribution of extragalactic X-ray emitting objects to the XRB in the $3-100 \mathrm{keV}$ energy interval is small so as not to substantially deform the optically thin thermal bremsstrahlung shape which is the prima facie evidence in support of the existence of a hot IGG from which the bulk of the XRB originates. How large this contribution is will be the subject of the next section.

\section{DISCRETE SOURCE CONTRIBUTION TO THE XRB}

Recent, detailed discussions on the contribution of known classes of extragalactic X-ray sources to the XRB can be found in Schmidt and Green (1986), Giacconi and Zamorani (1987), and Setti (1987). Since the various classes of objects are characterized by different spectral types, and therefore their percentage contributions to the XRB in general depend on the photon energy, it is useful for the sake of discussion to choose a reference photon energy for normalization. Since most data have been collected by the Einstein Observatory, which was sensitive in the energy range $0.3-3.5 \mathrm{keV}$, and since the XRB spectrum and the bright source sample spectra were observed at energies $>2 \mathrm{keV}$ by instruments such as HEAO- 1 and EXOSAT, it has become convenient to refer the estimates to an (observed) energy of $2 \mathrm{keV}$ where the intensity of the "reference" XRB is obtained by smoothly extrapolating the bremsstrahlung spectrum applicable to energies $>3 \mathrm{keV}$ downward with a power law of index -0.4 .

The estimates of the integrated contributions to the $2 \mathrm{keV} \mathrm{XRB} \mathrm{thus} \mathrm{defined} \mathrm{from} \mathrm{known}$ classes of sources are summarized in Table 1, following the discussion of Setti (1987). It should be noted that the contribution from the resolved sources down to the Einstein 


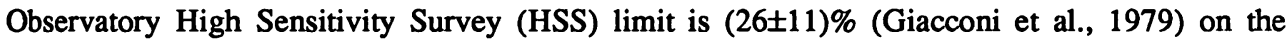
assumption that the energy spectra of the sources in the observed band are represented by a power law of index -0.5 . If, as will be discussed later, the spectral index is $\alpha \leq-1.0$, then the HSS limit should be reduced by $\sim 40 \%$.

TABLE 1. The Contribution of Known Classes of Discrete Extragalactic Sources to the $2 \mathrm{keV}$ XRB

\begin{tabular}{lcll}
\hline Objects & $\%$ & \multicolumn{2}{l}{ Remarks and References } \\
\hline Quasars & $8^{a}-13$ & $M_{B}<-23^{b}$ & Fitting total \# found in MSS survey. ${ }^{1}$ \\
& 20 & $M_{B}<-23.8$ & From the optical counts. \\
& $7^{a}-11$ & $M_{B}<-23.8$ & Same as above but fitting the MSS survey. ${ }^{2}$ \\
Seyfert 1 & 29 & $M_{B}>-23 \quad$ From HEAO-1 survey. No evolution. ${ }^{1,3}$ \\
& $>34$ & $M_{B}>-23.8$ Some evolution. ${ }^{2,4}$
\end{tabular}

- Percentage values obtained from Einstein Observatory data by adopting a steep spectral index $(\alpha=-1.2)$.

${ }^{b}$ Quoted $M_{B}$ values assume $H_{0}=50 \mathrm{kms}^{-1} \mathrm{Mpc}^{-1}$.

References: (1) Schmidt and Green (1986); (2) Setti (1987); (3) Piccinotti et al. (1982); (4) Setti and Woltjer (1982); (5) Giacconi and Zamorani (1987).

The dominant contribution apparently comes from AGNs (quasars and Seyfert type 1 nuclei), but the estimates are still affected by uncertainties.

\subsection{Quasars}

The highest percentage contribution reported in Table $1(\sim 20 \%)$ has been obtained by integrating over the X-ray source counts derived from the optical counts via the distribution of $\mathrm{X}$-ray to optical flux ratios provided by the observations of the Bright Quasar Survey (BQS) sample with the Einstein Observatory (Tananbaum et al., 1986). While the slope of the X-ray source counts thus derived agrees with that of the Einstein Observatory Medium Sensitivity Survey (MSS; Gioia et al., 1984) in the region of overlap their number exceeds by at least a factor of 1.6 that of the MSS (see Setti, 1987, for a detailed discussion). The reason(s) for this discrepancy is unknown. If the MSS counts are enforced, then one obtains a lower percentage value that is in very good agreement with that found by Schmidt and Green (1986). This is reassuring since the two estimates have been made by following completely different methodologies.

The spectra of quasars in the Einstein Observatory band $(0.2-3.5 \mathrm{keV})$ have been the subject of recent studies (Wilkes and Elvis, 1987; Canizares and White, 1989). The results are summarized in Table 2 . Obviously there is a marked difference between the flat radio spectral quasars (generally associated with powerful compact radio sources) and the radio-quiet quasars, with the steep radio spectra quasars (generally associated with powerful extended radio sources) providing some sort of intermediate case. It should be noted that the RL(F) quasars have an average spectral index which matches that of the XRB in the $3-10 \mathrm{keV}$ interval. By splitting their sample in redshift bins, Canizares and White (1989) do not find any indication of a redshift dependence of the average spectral indices, although the statistics are really good 
TABLE 2. X-ray Spectra of Quasars and Seyfert Type 1 Nuclei

\begin{tabular}{|c|c|c|c|c|}
\hline \multirow[b]{2}{*}{$\begin{array}{l}\text { Energy } \\
\text { Interval }\end{array}$} & \multirow[b]{2}{*}{$\begin{array}{l}\text { Quasar } \\
\text { Class }\end{array}$} & \multicolumn{3}{|c|}{$\langle\alpha\rangle$} \\
\hline & & $\begin{array}{c}\text { Wilkes and Elvis } \\
(1987)\end{array}$ & $\begin{array}{c}\text { Canizares and White } \\
\text { (1989) }\end{array}$ & $\begin{array}{c}\text { Tumer and Pounds } \\
\text { (1989) }\end{array}$ \\
\hline $0.2-3.5 \mathrm{keV}^{a}$ & $\begin{array}{l}\text { Radio-Loud (flat) } \\
\text { Radio-Loud (steep) } \\
\text { Radio-Quiet }\end{array}$ & $\begin{array}{l}-0.42 \pm 0.09 \\
-0.58 \pm 0.17 \\
-0.89 \pm 0.09^{b}\end{array}$ & $\begin{array}{l}-0.39 \pm 0.07 \\
-0.73 \pm 0.95 \\
-1.14 \pm 0.08\end{array}$ & \\
\hline $2-10 \mathrm{keV}^{c}$ & & & & $-0.70^{d}$ \\
\hline
\end{tabular}

a Average spectral indices for optical and radio selected samples of quasars. The differential energy spectra of the objects have been fitted with power laws of the form Flux $\propto \mathrm{E}^{\alpha}$.

b This sample contains several objects with $M_{B}>-23.8$. If only brighter quasars are considered then $\langle\alpha\rangle \cong-1.02$.

c A sample of 48 hard X-ray selected, mostly Seyfert type 1 nuclei, from EXOSAT observations.

$d$ With a dispersion $\sigma=0.17$. Available spectra for a small sample of quasars agrees with this average slope (see text).

enough only for the $R L(F)$ quasar sample showing that these objects maintain a flat X-ray spectrum up to an energy of $\sim 10 \mathrm{keV}$.

A statistical analysis of a fainter sample of X-ray selected AGNs from the Einstein Observatory Extended MSS yields an average spectral index $\langle\infty\rangle=-1.0$ (Maccacaro et al., 1988), like for the bright sample of optically selected AGNs of Wilkes and Elvis (1987), and therefore no indication of spectral evolution is found.

From the standpoint of the contribution to the $2 \mathrm{keV} \mathrm{XRB}$ it is important to note that the Einstein Observatory observations have usually been reduced by assuming a spectral slope of -0.5 , while if the actual slope is $\leq-1.0$ (as appears to be the case for the RQ samples of Table 2) then the corresponding $2 \mathrm{keV}$ monochromatic fluxes should be reduced by $~-40 \%$ (Tananbaum et al., 1986). Since the great majority of the quasar population is composed of RQ objects ( $290 \%)$, then the lowest estimates reported in Table 2 are found.

Keeping in mind that RL quasars are on average stronger $X$-ray sources than the RQ quasars ( a factor 3 after Zamorani et al., 1981) one can conclude that the contribution of quasars to the $2 \mathrm{keV}$ XRB has an upper bound of $14 \%$ and could be as low as $8 \%$ if the MSS counts are matched.

The hard X-ray spectra of quasars are poorly known. Only very few had been measured until recently, notably 3C 273 with $\alpha \leq-0.05$ in the $2-30 \mathrm{keV}$ interval (Worrall et al., 1979), but the sample is now growing as a result of the data which are being obtained by the instruments on board the Japanese X-ray satellite GINGA in the $2-30 \mathrm{keV}$ energy interval. The fitted power law spectra are consistent with an average slope and a dispersion typical of Seyfert type 1 nuclei to be discussed next (Inoue, 1989).

\subsection{Seyfert 1 Nuclei (or Low Luminosity AGNs)}

A minimal contribution of $29.0 \pm 6.5 \%$ is obtained from the HEAO-1 A2 complete sample of bright sources and uniform space distribution, but this figure may be substantially increased by the presence of cosmological evolution for which there is some indication at the bright end of the Seyfert type 1 luminosity function (Setti, 1984). If the integral optical counts of AGNs were to continue with the slope $\cong 1$, applicable to the B magnitude interval $21-23$, down to $\mathrm{B} \cong 27-28$, then most of the $2 \mathrm{keV}$ XRB would originate in low luminosity AGNs at redshifts $z \sim 1-2$ (Setti and Woltjer, 1982; Setti, 1987; Giacconi and Zamorani, 1987; Anderson 
1987; Hamilton and Helfand, 1987). This would correspond to extending the X-ray source counts down to a flux $\sim 100$ times fainter than the HSS limit with a correspondingly flat slope consistent with the arcminute scale fluctuation analysis of Hamilton and Helfand (1987).

As is well known, however, the main problem resides in the discrepancy between the average spectrum of this type of sources and that of the XRB. A sizable sample of bright nearby hard X-ray selected Seyfert type 1 nuclei has been measured with the detectors on board EXOSAT in the energy interval $0.1-10 \mathrm{keV}$ (Turner and Pounds, 1989). In the 2-10 $\mathrm{keV}$ interval the spectra are well described by single power laws with a mean spectral index $\langle\alpha\rangle=-0.7$ and a dispersion $\sigma=0.17$ around the mean, in good agreement with previous findings based on HEAO-1 A2 data (Mushotzky, 1984). It is interesting to note that the soft $\mathrm{X}$-ray spectra of this hard X-ray selected sample are complex but include several which show a turn up of the spectrum at $\sim 1 \mathrm{keV}$ in agreement with the average steep slope found in the Einstein Observatory band for corresponding low luminosity AGNs (Wilkes and Elvis, 1987).

Thus, if the AGNs were to supply the bulk of the XRB, then the relevant portions of their spectra should be much flatter $(\langle\alpha\rangle \geq-0.4)$ than measured in the nearby sample, which implies the presence of a very significant cosmological evolution (e.g., Morisawa and Takahara, 1989, for recent modeling). In fact, it has been shown (Danese et al., 1986) that any population with hard X-ray spectra typical of the nearby AGN sample cannot contribute more than $\sim 30 \%$ to the total XRB energy flux in the $2-10 \mathrm{keV}$ interval; otherwise, it would produce spectral wiggles incompatible with the general smoothness of the XRB spectrum (De Zotti et al., 1982).

Now some brief notes concerning the remaining classes of sources in Table 1.

\subsection{Clusters of Galaxies}

Their emission is due to optically thin thermal bremsstrahlung of hot intracluster gas with typical temperature $\sim 6 \mathrm{keV}$, much below that required for the XRB. The estimate of the contribution is based on the HEAO-1 A2 survey, without including possible cosmological evolution scenarios which may enhance the expected contribution.

\subsection{Normal Galaxies}

Very little is known about their hard X-ray spectra (Makishima and Ohashi, 1989). Their shapes will depend in general on the particular admixture of galactic sources making up their global emission. The resulting spectra could also be rather flat. In any case normal galaxies and clusters together are bound to make a non-negligible contribution to the low energy part of the XRB spectrum.

\subsection{Other Sources}

Here we have included the BL Lacs, whose contribution is still uncertain but most likely not more than a few percentage points, radio galaxies and other types of active galaxies. BL Lacs are also known to have rather steep spectra above $2 \mathrm{keV}$. 


\section{THE "RESIDUAL" XRB}

If the spectra of the AGNs are on average as steep as those measured in the bright sample, then the discrete source contributions to the XRB above $3 \mathrm{keV}$ can be subtracted out and one is left with the problem of explaining the "residual" background which still incorporates most of the energy flux (Leiter and Boldt, 1982; Setti, 1985; Giacconi and Zamorani, 1987). The low energy side of the residual XRB becomes very flat up to $20-30 \mathrm{keV}$ (spectral index $\alpha \geq-0.2$ below $\sim 10 \mathrm{keV}$ ), the precise shape depending on the spectral distribution and percentage contributions of the source populations to be subtracted. Note that, as we have previously discussed, the contribution of the AGNs to the $2 \mathrm{keV} \mathrm{XRB} \mathrm{is} \mathrm{uncertain} \mathrm{within} \mathrm{at} \mathrm{least} \mathrm{a}$ factor of two. Giacconi and Zamorani (1987) have modeled the residual XRB by making different assumptions on the relevant quantities, including "minimal" assumptions. The main conclusions one can draw are:

(a) The residual XRB can no longer be fitted by an optically thin thermal bremsstrahlung with a temperature of $-40 \mathrm{keV}$. Even the "minimal" contribution of the discrete sources destroys its extremely good representation of the observed spectrum in the 3 $100 \mathrm{keV}$ range (in a way it is too good!), so that no physical meaning can be attached to it.

(b) To explain the residual XRB one needs to postulate a new class of sources since the spectral characteristics are unlike those found in the known classes of powerful extragalactic X-ray emitters. For instance, this has led Leiter and Boldt (1982) to propose that such sources could be found in precursor AGNs, where gas accretion onto a central supermassive black hole first generates a very compact, hot, slightly comptonized thermal source prevalently emitting hard X-rays (luminosity $L \sim 1 / 10 L_{E}$, with $L_{E}$ the Eddington luminosity), which then evolves to a less compact Seyfert type nucleus $\left(L \sim 1 / 100 L_{E}\right)$ in a characteristic time interval corresponding to the Hubble time at $z \sim 3$ (Boldt and Leiter, 1987). These authors have assumed a total contribution from known extragalactic X-ray objects $(\sim 50 \%$ at $2 \mathrm{keV})$ such that the residual XRB would have a spectral slope $\alpha=-0.2$ at the low energy end. This model provides in a way a somewhat extreme example of the spectral evolution for AGNs required to explain the XRB.

The extreme flatness of the residual XRB spectrum excludes the possibility that it is due to an optically thin bremsstrahlung from a hot diffuse gas. The amount of comptonization required to flatten the spectrum leads in fact to rather stringent upper limits on the size of the thermally emitting regions, and one falls back into the concept of compact sources as discussed in the preceding paragraph (Giacconi and Zamorani, 1987).

If the total contributions of known classes of extragalactic sources turn out to be a very large fraction of the $2 \mathrm{keV} \mathrm{XRB}$, then one would be led to a rather intriguing situation, since it is not obvious what kind of sources and/or physical processes and space distribution could provide both the intensity and the very flat $(\alpha \cong 0.0)$, or even convex (positive slope at a few $\mathrm{keV}$ ) spectrum of the ensuing residual XRB (Setti, 1985).

\section{ALTERNATIVE MODELS}

In addition to the models already discussed, many others have been proposed in the attempt to explain the XRB in the course of almost three decades after its discovery (Giacconi et al., 
1962). It is obviously impossible to summarize here all the work which has been done. We shall limit ourselves to briefly outlining some other proposals put forward in this decade to give a flavor of the ample possibilities which remain open. These ideas are generally based on assumptions concerning the evolution of the physical properties of normal galaxies or on the physics of AGNs and their cosmological evolution or on cosmological events of some form, all of which are in general difficult to verify observationally (as it should be for any long-standing hypothesis!).

Bookbinder et al. (1980) have made the interesting conjecture that the XRB can be essentially supplied by the thermal bremsstrahlung emission associated with hot galactic winds powered by an increased rate of supernovae in young galaxies. This requires that on average each galaxy produces $\geq 10^{10}$ supernovae over a typical time interval of $10^{7}$ years and that the epoch of galaxy formation is rather recent, $z \cong 2$, otherwise the temperature of the wind would not be high enough to cope with the redshift effect. There are other difficulties with this model: Giacconi and Zamorani (1987) noted that the associated optical emission would be such that the surface density of young galaxies at faint optical magnitudes would be much larger than what has actually been found, while it is also likely that the $\gamma$-ray emission associated with these energetic events would produce a $\gamma$-ray background flux far in excess of the observed one (Setti and Woltjer, 1982). These authors also discussed the possible contribution of massive X-ray binaries whose spectra are known to be flat, much like that of the XRB, and to extend up to $\sim 20 \mathrm{keV}$. This possibility has been revived by Griffiths (1989, and poster paper), who argues that the integrated emission from an early population of low metallicity massive X-ray binaries in star-forming galaxies may contribute up to $\sim 50 \%$ of the XRB in the $3-20 \mathrm{keV}$ energy interval if most of the contributed flux originates in galaxies with redshifts confined to $z<1$.

Daly (1987) has proposed a unified picture in which the XRB, the formation of galaxies and voids are all parts of the same scenario. The XRB would be produced by thermal bremsstrahlung emission associated with large $\left(10^{15-16} M_{\odot}\right)$ gravitationally bound condensates of matter whose formation and successive evolution are governed by the properties of an (unspecified) unstable dark matter particle candidate. The main difficulty here, at least as far as the origin of the XRB is concerned, is that the surface density of the condensates is much smaller than the lower bound imposed by the XRB fluctuations, unless, as the author argues, the condensates are constrained to form following a very regular pattern.

That the XRB could be linked to the formation of structures in the universe has also been proposed by Ostriker et al. (1986) in a different scenario, where superconducting cosmic strings are assumed to release enormous amounts of energy to the surrounding intergalactic medium, thereby producing huge expanding shells of hot gas on the edge of which galaxies could form after cooling. This picture predicts the existence of a very hot intergalactic gas with a bubblelike structure and temperatures of $10^{8}-10^{9} \mathrm{~K}$ which may contribute to the $\mathrm{X}$-ray background.

Fabian et al. (1988) propose that the hard X-ray shape of the XRB spectrum can be explained by a nonthermal process in which the acceleration mechanism of the electrons is "loaded" by the electron-positron pairs created in photon-photon collisions. This feedback mechanism would "naturally" produce a sharp break in the emitted photon spectrum at the electron rest mass energy, much larger however than the observed $\sim 40 \mathrm{keV}$. As a result these hypothetical sources, identified as young active galaxies, should be placed at redshifts $\mathrm{x} \cong$ $10-30$, emitting on average $\sim 10^{46} \mathrm{erg} \mathrm{s}^{-1}$ each.

Another possibility is proposed by Daly (1989). It is assumed that most bright galaxies possessed a relatively massive black hole $\left(-10^{7} \mathrm{M} \odot\right)$ at an early stage of their formation 
(redshift $z \approx 2.5$ ) and that they went through a phase lasting for $\sim 3 \times 10^{7} \mathrm{yr}$ in which highly collimated jets departing from the central source with speeds of a few $\times 10^{3} \mathrm{~km} \mathrm{~s}^{-1}$ would channel through and shock-heat the surrounding interstellar gas to temperatures of $\sim 200 \mathrm{keV}$. On average the X-ray luminosities would be $\sim 10^{45} \mathrm{erg} \mathrm{s}^{-1}$. In a way this model resembles that of the "precursor" AGNs of Leiter and Boldt (1982), but the detailed mechanisms are different.

Finally, let me briefly introduce a new scenario (Setti and Wolfjer, 1989) which basically follows from a unified scheme of AGNs recently proposed (Barthel, 1989). According to this scheme the RL quasars and the strong radio galaxies are members of the same population, the RL quasar phenomenon showing up whenever the associated relativistic beams are aimed toward us within a certain angle from the line of sight. Similarly, it is proposed that the radio-quiet quasars and luminous infrared galaxies can be unified, the apparent morphological differences being attributable only to the geometrical orientation of the sources with respect to the observer. In this framework one has to assume that quasars and Seyfert type 1 nuclei remain hidden by optically thick torii of surrounding absorbing material whenever the line of sight is not favorably placed. Setti and Woltjer (1989) show that with very reasonable assumptions the X-rays emitted by the central sources can also be effectively absorbed up to $30 \mathrm{keV}$, or more, which can explain why quasars and Seyfert type 1 nuclei are observed to be much stronger X-ray sources than radio galaxies and IR galaxies in the Einstein Observatory energy band. At energies $\geq 10 \mathrm{keV}$, depending on the precise value of the absorbing column of gas along the line of sight, the radio galaxies and IR galaxies would have to show the same hard $\mathrm{X}$-ray spectra as quasars and Seyfert type 1 nuclei if Barthel's unified picture holds. It is inferred that by assuming an appropriate distribution of the absorption cut-offs combined with a "mild" cosmological evolution of the global AGNs population up to a redshift $z \cong 2.5-3$, one can account for the overall XRB even if the average hard X-ray spectrum of the sources is as steep as that observed in the bright sample of Seyfert type 1 nuclei.

\section{CONCLUDING REMARKS}

If on the one hand the broad astrophysical and cosmological implications involved in the extragalactic XRB justify the continuing interest in its study, on the other hand the variety of models which have been and are being proposed is in itself a demonstration of the need for new and deeper observations in order to constrain the various possibilities. As a result of our discussion it is clear that the basic problem still remains the determination of the contribution of known classes of extragalactic sources, in particular AGNs, over the energy range of interest for the XRB. In fact, most papers dealing with the XRB terminate by deferring the solution to the new and more sensitive X-ray missions now being developed. It is hoped, but by no means certain, that this aim will be achieved.

Before closing, let me make a few comments on the extragalactic $\gamma$-ray background (see Ramana Murthy and Wolfendale, 1986, for a more comprehensive discussion). The main problem here is that only a few extragalactic objects have been detected at $\gamma$-ray energies, but interestingly enough they can already provide some useful insight into the problem of the origin of the $\gamma$-ray background. Thus it has been shown (Schönfelder, 1978) that $\sim 10 \%$ of the Seyfert type 1 galaxies emitting at the level of NGC 4151 would saturate the $\sim 2 \mathrm{MeV}$ background. Likewise no more than $\sim 10 \%$ of quasars can have emission properties similar to $3 \mathrm{C}$ 273 , otherwise the background intensity at $\sim 100 \mathrm{MeV}$ would be exceeded (Setti and Woltjer, 1979). The integrated contribution from normal galaxies may also provide a large fraction of 
the $\sim 100 \mathrm{MeV}$ background, depending on the galaxy content of cosmic rays and interstellar gas and its past history. So it appears reasonable that the extragalactic $\gamma$-ray background from -1 to $\sim 200 \mathrm{MeV}$ can be explained in terms of unresolved sources (e.g., Bignami et al., 1979). It is hoped that extragalactic $\gamma$-ray astronomy will be boosted out of its presently uncertain observational status with the coming into operation of the new dedicated space missions, such as GRO and SIGMA.

\section{REFERENCES}

Anderson, S. F., and Margon, B. 1987, Astrophys. J., 314, 111.

Barcons, X. 1987, Astrophys. J., 313, 547.

Barcons, X. 1989, in Proc. IAU 139, Galactic and Extragalactic Background Radiation, ed. S. Bowyer, and Ch. Leinert (Dordrecht: Kluwer Academic Publishers).

Barcons, X., and Fabian, A. C. 1988, Mon. Not. R. astr. Soc., 230, 189.

Barcons, X., and Lapiedra, R. 1985, Astrophys. J., 289, 33.

Barthel, P. D. 1989, Astrophys. J., 336, 606.

Bignami, G. F., Fichtel, C. E., Hartmann, R. F., and Thompson, D. J. 1979, Astrophys. J., 232, 649.

Boesgaard, A. M., and Steigman, G. 1985, Ann. Rev. Astr. Astrophys., 23, 319.

Boldt, E. 1987, Phy. Reports, 146, No. 4, 215.

Boldt, E. 1988, NASA Report \#88-037.

Boldt, E., and Leiter, D. 1987, Astrophys. J., 322, L1.

Bookbinder, J., Cowie, L. L., Krolik, J. H., Ostriker, J. P., and Rees, M. J. 1980, Astrophys. J., 237, 647.

Canizares, C. R., and White, J. L. 1989, Astrophys. J., 339, 27.

Cheng, E. S., Saulson, P. R., Wilkinson, D. T., and Corey, B. E. 1979, Astrophys. J., 232, L139.

Cowsik, R., and Kobetich, E. J. 1972, Astrophys. J., 177, 585.

Daly, R. A. 1987, Astrophys. J., 322, 20.

Daly, R. A. 1989, paper presented at IAU Symposium 139, "Galactic and Extragalactic Background Radiation,' June 12-16, 1989, Heidelberg, Federal Republic of Germany.

Danese, L., De Zotti, G., Fasano, G., and Franceschini, A. 1986, Astrophys. J., 161, 1.

De Zotti, G., Boldt, E. A., Cavaliere, A., Danese, L., Franceschini, A., Marshall, F. E., Swank, J. H., and Szymkowiak, A. E. 1982, Astrophys. J., 253, 47.

Fabian, A. C., Done, C., and Ghisellini, G. 1988, Mon. Not. R. astr. Soc., 232, 21P.

Fichtel, C. E., Simpson, G. A., and Thompson, D. J. 1978, Astrophys. J., 222, 833.

Field, G. B., and Perrenod, S. C. 1977, Astrophys. J., 215, 717.

Fomalont, E. B., Kellerman, K. I., and Wall, J. V. 1984. Astrophys. J., 277, L23.

Giacconi, R., Gursky, H., Paolini, F., and Rossi, B. 1962, Phys. Rev. Letters, 9, 439.

Giacconi, R., et al. 1979, Astrophys. J., 234, L1.

Giacconi, R., and Zamorani, G. 1987. Astrophys. J., 313, 20.

Gioia, I. M., Maccacaro, T., Schild, R. E., Stocke, J. T., Liebert, J. W., Danziger, I. J., Kunth, D., and Lub, J. 1984, Astrophys. J., 283, 495.

Griffiths, R. E. 1989, NATO-ASI on "The Epoch of Galaxy Formation," eds. C. S. Frenk et al. (Kluwer, Dordrecht), 235.

Gruber, D. E., Rothschild, R. E., Matteson, J. L., and Kinzer, R. L. 1984, in X-Ray and UV Emission from Active Galactic Nuclei, eds. W. Brinkmann and J. Trümper, MPE Report 184, 129.

Guilbert, P. W., and Fabian, A. C. 1986, Mon. Not. R. astr. Soc., 220, 439.

Hamilton, T. T., and Helfand, D. J. 1987, Astrophys. J., 318, 93.

Inoue, H. 1989, in Big Bang, Active Galactic Nuclei and Supernovae, Universal Ac. Press, Tokyo, 301.

Kinzer, R. L., Johnson, W. N., and Kurfers, J. D. 1978, Astrophys. J., 222, 370.

Knoke, J. E., Partridge, R. B., Ratner, M. I., and Shapiro, I. I. Astrophys. J., 284, 479.

Leiter, D., and Boldt, E. 1982, Astrophys. J., 260, 1.

Maccacaro, T., Gioia, I. M., Wolter, A., Zamorani, G., and Stocke, J. T. 1988, Astrophys. J., 326, 680.

Makishima, K., and Ohashi, T. 1989, in Big Bang, Active Galactic Nuclei and Supernovae, Universal Ac. Press, Tokyo, 371.

Marshall, F. E., Boldt, E. A., Holt, S. S., Miller, R. B., Mushotzky, R. F., Rose, L. A., Rothschild, R. E., and Serlemitsos, P. J. 1980, Astrophys. J., 235, 4. 
Morisawa, K., and Takahara, F. 1989, P. A. S. J., to be published.

Mushotzky, R. F. 1984, in COSPAR/IAU Symposium, High-Energy Astrophysics and Cosmology, eds. G. F. Bignami and R. A. Sunayev, Adv. Space Res., 3, 157.

Ostriker, J. P., Thompson, C., and Witten, E. 1986, Phys. Letters, B180, 231.

Piccinotti et al. 1982, Astrophys. J., 253, 485.

Ramana Murthy, P. V., and Wolfendale, A. W. 1986, Gamma Ray Astronomy (Cambridge University Press, Cambridge).

Reeves, H., Richer, J., Sato, K, and Terasawa, N. 1989, preprint.

Schmidt, M., and Green R. F. 1986, Astrophys. J., 305, 68.

Schönfelder, V. 1978, Nature, 274, 344.

Schönfelder, V., Grami, F., and Penningsfeld, F.-P. 1980, Astrophys. J., 240, 350.

Setti, G., and Woltjer, L. 1979, Astron. Astrophys., 76, L1.

Setti, G., and Woltjer, L. 1982, in Astrophysical Cosmology, eds. H. A. Bruck, G. V. Coyne, and M. S. Longair (Pontificia Academia Scientiarum, Vatican City), 315.

Setti, G. 1984, in X-Ray and UV Emission from Active Galactic Nuclei, eds. W. Brinkmann and J. Trümper, MPE Report 184, 243.

Setti, G. 1985, in Non-Thermal and Very High Temperature Phenomena in X-Ray Astronomy, eds. G. C. Perola and M. Salvati (Istituto Astronomico, Università "La Sapienza," Rome), 159.

Setti, G. 1987, in IAU Symposium No. 124, Observational Cosmology, eds. A. Hewitt, G. Burbidge, and L. Z. Fang (Reidel, Dordrecht), 579.

Setti, G., and Woltjer, L. 1989, submitted.

Shafer, R. A. 1983, NASA Tech. Mem. 85029.

Shafer, R. A., and Fabian, A. C. 1983, in IAU Symposium No. 104, Early Evolution of the Universe and Its Present Structure, eds G. O. Abell and G. Chincarini (Reidel, Dordrecht), 333.

Smoot, G. F., Gorenstein, M. V., and Muller, R. A. 1977, Phys. Rev. Letters, 39, 898.

Tananbaum, H., Avni, Y., Green, R. F., Schmidt, M., and Zamorani, G. 1986, Astrophys. J., 305, 57.

Taylor, G. B., and Wright, E. L. 1989, Astrophys. J., 339, 619 .

Thompson, D. J., and Fichtel, C. E. 1982. Astron. Astrophys., 109, 352.

Trombka, J. I, Dyer, C. S., Evans, L. G., Bielefeld, M. J., Seltzer, S. M., and Metzger, A. E. 1977, Astrophys. J., 212, 925.

Turner, T. J., and Pounds, K. A. 1989. Mon. Not. R. astr. Soc., submitted.

Tyson, J. A. 1984, in IAU Colloquium 78, Astronomy with Schmidt Type Telescopes, ed. M. Capaccioli (Reidel, Dordrecht), 489.

Wilkes, B. J., and Elvis, M. 1987, Astrophys. J., 323, 243.

Worrall, D. M., Mushotzky, R. F., Boldt, E. A., Holt, S. S., and Serlemitsos, P. J. 1979, Astrophys. J., 232, 683 .

Zamorani, G., et al. 1981, Astrophys. J., 245, 357.

M. Lampton: Your statistical absorption model would predict about half the $30-100 \mathrm{keV}$ extragalactic sources would show X-ray absorption. Is this observed?

G. Setti: There are indeed observational indications of drift absorption in active galactic nuclei. However, to absorb up to $30 \mathrm{keV}$ would require a column density of $\approx 10^{25} \mathrm{~cm}^{-2}$, which can be provided by a toroidal configuration with a size of several $\mathrm{pc}$ and a total mass of $\approx 10^{6} M_{\odot}$. This does not seem unreasonable.

Biermann: There is a paper reporting GINGA High energy X-ray results on the Seyfert 1 galaxies N4051 and MCG-6-30-15 (Matsuoka et al., preprint) confirming such column densities through X-ray absorption. 